\title{
Non-radiative energy-transfer-driven quantum dot LEDs
}

\author{
Burak Guzelturk $^{1}$, Talha Erdem ${ }^{1}$, Emre Unal ${ }^{1}$, Sedat Nizamoglu ${ }^{1}$, Donus Tuncel ${ }^{1}$, and \\ Hilmi Volkan Demir ${ }^{1,2^{*}}$ \\ ${ }^{1}$ Department of Electrical and Electronics Engineering, Department of Physics, Department of Chemistry, and UNAM - Institute of \\ Materials Science and Nanotechnology, Bilkent University, TR-06800, Ankara, Turkey; ${ }^{2}$ Nanyang Technological University, School of \\ Electrical \& Electronic Engineering, Division of Microelectronics, School of Mathematical and Physical Sciences, Luminous! \\ Semiconductor Lighting and Display Center of Excellence, Nanyang Avenue, Singapore 639798, Singapore \\ *Tel:[+90]( 312) 290 1021, e-mail: volkan@bilkent.edu.tr
}

Semiconductor nanocrystal quantum dots with their tunable optical properties, narrow photoluminescence, and high photostability have generated great interest in light-emitting device applications. [1,2] Such colloidal quantum dots that are directly electrically driven in light-emitting diode (LED) structures have been extensively studied in the last decade. However, these LEDs unfortunately suffer from the fundamental problem of poor charge injection into these nanocrystals, which have high potential barriers due to their ligands surrounding them. This problem has been investigated and attempted to be minimized through various optimization methods. $[3,4]$ As a result, although today the device external quantum efficiencies can now reach ca $2.7 \%$ [5], the main problem with charge injection still exists. On the other hand, conjugated conductive polymers are being used for organic based LEDs for several decades. These LEDs can reach higher quantum efficiencies over $20 \%$ [6]. But these devices suffer in most of the cases from instabilities and degradation of the polymer active layers. To combine the advantages of each material system, hybrid organic-inorganic structures have also previously been investigated to obtain more ingenious devices. However, in all of these hybrid devices, the basic operation has mainly been based on the same mechanism of conventional charge injection into the nanocrystal active material.

In this study, as a paradigm shift, we propose and demonstrate non-radiative energy-transfer-driven quantum dot LEDs that inject excitons into the active nanocrystal material, instead of injecting charges into them. For that we hybridize specially functionalized conjugated polymers to be attached to the colloidal quantum dots to drive them in the hybrid LED structure via Förster-type non-radiative energy transfer from the polymers to the dots when the LED is electrically biased. Under electrical injection, these quantum dots are therefore not directly electrically excited but through non-radiative resonance energy transfer from conjugated polymer donors that are electrically driven. This method can be an alternative route for quantum dot LEDs with possible prospects of higher external quantum efficiencies in the case of using properly chosen host polymer and transport layers. As a result highly efficient quantum dot based light emitting devices can be implemented using non-radiative energy transfer. To make a proof-of-concept demonstration, we utilize a simple device architecture that consists of glass/transparent oxide (ITO) /active layer /aluminum top contact, where the active layer is the hybrid composite of CdSe/ZnS QDs integrated with polyfluorene (PF) derivatives. In operation, PF is electrically driven, which transfers its excitons to QDs and these excited QDs in turn luminescence. Here we present electroluminescence from quantum dots via non-radiative energy transfer when the LED is electrically driven, along with a systematic study of optical characterizations that showed the strong evidence for energy transfer.
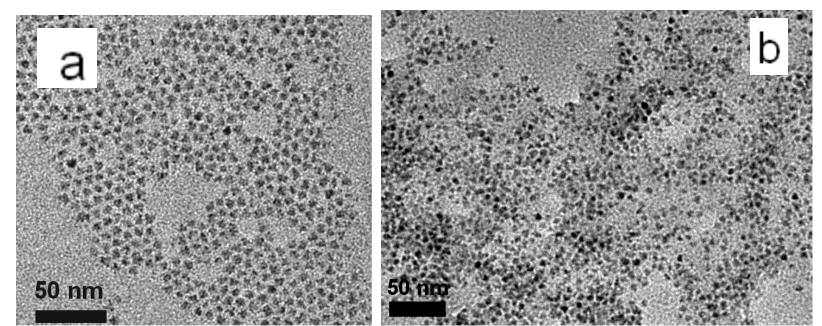

Figure 1. (a) Transmission electron microscopy (TEM) image of TOPO capped CdSe/ZnS blended with PF polymer and (b) TOPO capped $\mathrm{CdSe} / \mathrm{ZnS}$ hybridized with carboxyl functionalized PF. The scale bar is $50 \mathrm{~nm}$.

Several chemistry groups have previously successfully attached various quantum dots to polymers having larger surface binding affinity than the ligands of these quantum dots and demonstrated hybrid structures of polymers and quantum dots. $[7,8]$ For example, $\mathrm{CdSe} / \mathrm{ZnS}$ quantum dots have been integrated with different polyfluorene (PF) derivatives. In this work, we used blue emitting carboxyl group functionalized polyfluorene derivative conjugated polymer, which serve as the donors in the hybrid structure, and core/shell CdSe/ZnS quantum dots, which serve as the acceptors. Because of high spectral overlap of donor emission with the absorption of the acceptor, and the donor-acceptor species physically being in the close proximity of each other as a result of chemical binding, we achieved strong non-radiative energy transfer, with rates up to $88 \%$ so that emission of the polymer is strongly quenched and the emission of quantum dots is substantially enhanced. Fig. 1(a) shows the TOPO capped CdSe/ZnS quantum dots mechanically blended with PF polymer, which does not have specific binding group, and Fig.1(b) shows the hybrid film of carboxyl group functionalized PF with the same quantum dots. Carboxyl groups replace TOPO ligands and hang onto the quantum dots. Phase segregation problem is thus 
overcome by utilization of specific binding groups those are functionalized to polymer as it can be observed in Fig. 1. However, this also drops the quantum efficiency due to the decreased passivation of the quantum dots. Instead amine capped $\mathrm{CdSe} / \mathrm{ZnS}$ quantum dots, which can also specifically bind to carboxyl groups, provide better performance because the drop in the quantum efficiency is small. Photoluminescence (PL), PL excitation (PLE) and time-resolved PL measurements are systemically taken to understand the energy transfer dynamics of the hybrid composites. Different ratios of acceptors to donors (A/D) are investigated in Fig. 2. Here A/D is expressed as the ratio of the number of quantum dots to number of repeat units in polymer.
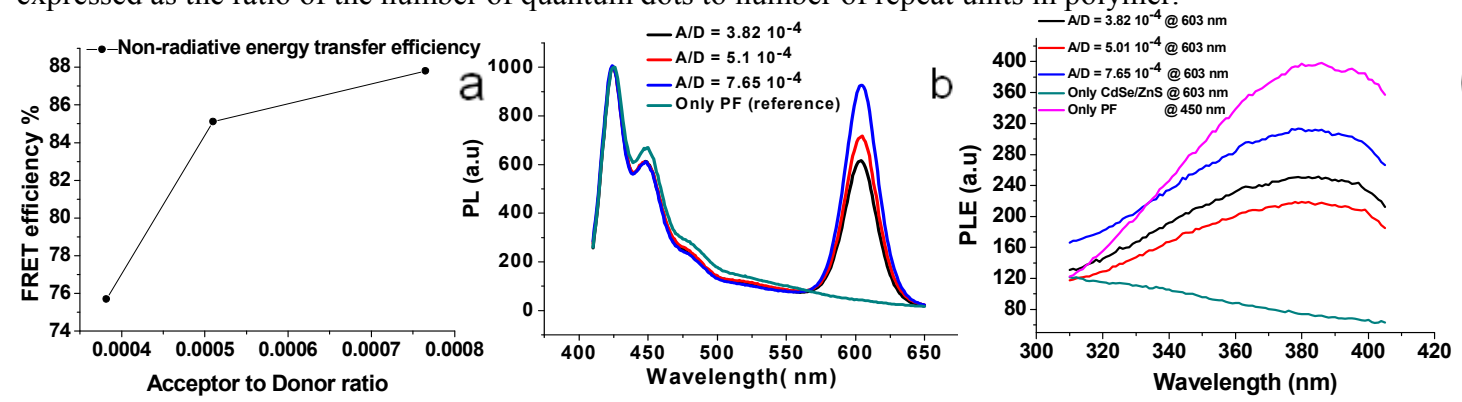

Figure 2. (a) Non-radiative energy transfer efficiency, (b) PL and (c) PLE of thin films of hybrid composites.

Only donor exhibits a photoluminescence lifetime of $74 \mathrm{ps}$, which is reduced to $18 \mathrm{ps}, 11 \mathrm{ps}$, and 9 ps as the A/D ratio is increased systemically. Non-radiative energy transfer rate is calculated from $1-\left(\tau_{\mathrm{DA}} / \tau_{\mathrm{D}}\right)$, where is $\tau_{\mathrm{DA}}$ is the lifetime of donors in the presence of acceptors and $\tau_{\mathrm{D}}$ is the lifetime of donors in the absence of acceptors. The maximum energy transfer rate is found to be $88 \%$ (Fig. 2(a)-(b)). Also, PLE (see Fig.2(c)) measurements of the hybrid films show that excitation of the quantum dots are almost the same as the excitation of the polymers, which indicates that emission of the quantum dots at $603 \mathrm{~nm}$ is a result of the excitation of PF donor polymers. Electrically driven devices include a simple, non-optimized device structure of glass / ITO / PEDOT:PSS / active layer / Al. Ideally, electron and hole transport layers (ETL and HTL) need to be included in the device structure to obtain balanced and enhanced charge injection in the polymer. Measured electroluminescence (EL), current vs. voltage (I-V), and output power vs. voltage (LI) characteristics of the fabricated devices are shown in Fig. 3.
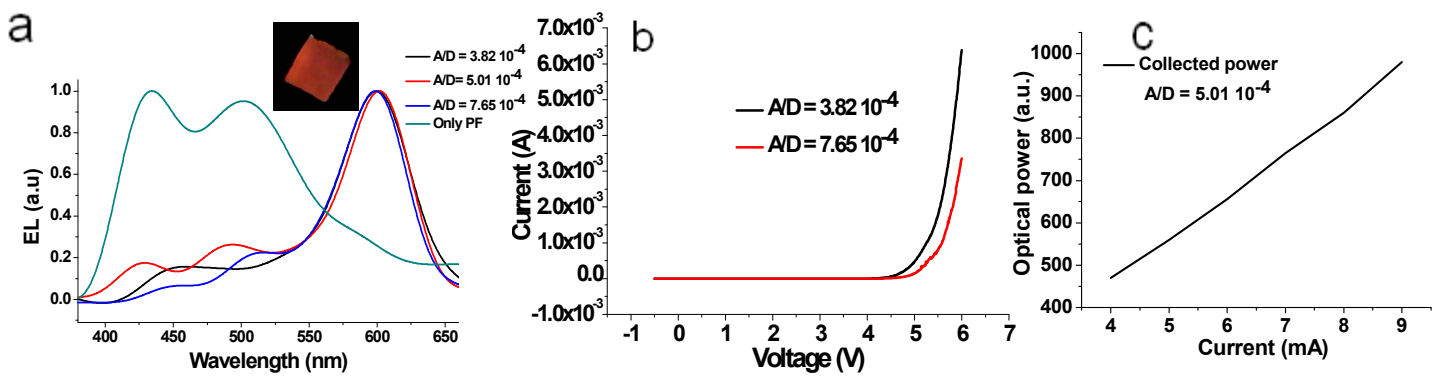

Figure 3. (a) EL, (b) I-V, (c) LI measurements of the devices.

Green emission from PF polymer under bias is attributed to the structural change of the polymer due to heating during contact evaporation. As the quantum dot ratio increases in the devices, stronger quantum dot EL is obtained because of increasing energy transfer rate. I-V characteristics reveal that turn-on voltage rises up as the quantum dot content is increased due to lowered conductivity of the active layer. The devices that include only quantum dot do not show any detectable EL due to poor charge injection. Luminous intensities of the hybrid devices that include polymer integrated QDs were on the order of microwatts. The absence of injection layers cause non-optimized charge injection into the active layer of the hybrid devices.

In this study we showed that non-radiative energy transfer between conjugated polymers and colloidal quantum dots can be efficiently achieved by functionalizing the polymers with specific groups. Driving the quantum dots via excitons instead of direct charge injection is more advantageous and efficient. Although this work showed a proof-of-concept demonstration, further optimizations of the hybrid LEDs are necessary so that highly efficient quantum dot LEDs can be obtained. Utilization of non-radiative energy transfer with high efficiencies overcomes the problems of poor charge injection into colloidal quantum dots.

This work is supported by NRF RF 2009-09, EU-FP7 Nanophotonics4Energy NoE, and TUBITAK EEEAG 107E088, 109E002, 109E004, and 110E010. HVD acknowledges support from ESF-EURYI and TUBA-GEBIP, and BG and TE from TUBITAK-BIDEB

References

[1] V.L. Colvin et al., Nature 370, 354, 1994

[2] Q. Sun et al. Nat. Photonics 1, 717, 2007

[3] S.Coe et al., Nature 420, 800, 2002

[4] J. Zhao et al., Nano Lett. 6, 463, 2006

[6] D. Tanaka et al., J. App. Phys. 46, L10, 2007

[7] M. Tamborra et al., Small 3, 822, 2007

[5] P.O. Anikeeva et al., Nano Lett. 9, 2532, 2009

[8] J. Kwak et al., Adv. Mater. 21, 5022, 2009 